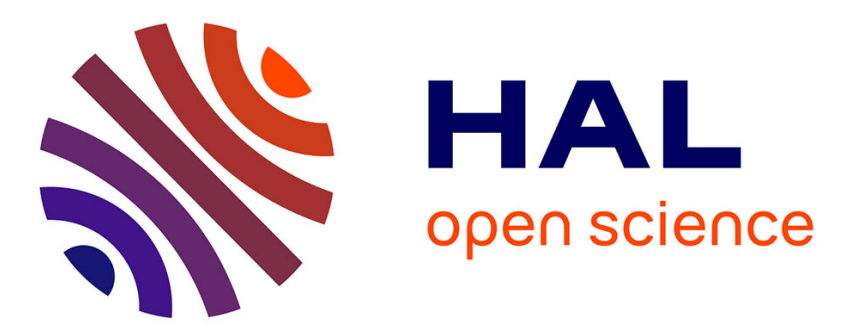

\title{
Introducing interactive inverse FEM simulation and its application for adaptive radiotherapy
}

Eulalie Coevoet, Nick Reynaert, Eric Lartigau, Luis Schiappacasse, Jérémie Dequidt, Christian Duriez

\section{- To cite this version:}

Eulalie Coevoet, Nick Reynaert, Eric Lartigau, Luis Schiappacasse, Jérémie Dequidt, et al.. Introducing interactive inverse FEM simulation and its application for adaptive radiotherapy. MICCAI - 17th International Conference on Medical Image Computing and Computer-Assisted Intervention, Sep 2014, Boston, United States. hal-01059667

\section{HAL Id: hal-01059667 https://inria.hal.science/hal-01059667}

Submitted on 1 Sep 2014

HAL is a multi-disciplinary open access archive for the deposit and dissemination of scientific research documents, whether they are published or not. The documents may come from teaching and research institutions in France or abroad, or from public or private research centers.
L'archive ouverte pluridisciplinaire HAL, est destinée au dépôt et à la diffusion de documents scientifiques de niveau recherche, publiés ou non, émanant des établissements d'enseignement et de recherche français ou étrangers, des laboratoires publics ou privés. 


\title{
Introducing interactive inverse FEM simulation and its application for adaptive radiotherapy
}

\author{
Eulalie Coevoet ${ }^{1,2}$, Nick Reynaert ${ }^{1}$, Eric Lartigau $^{1}$, Luis Schiappacasse $^{1}$, \\ Jérémie Dequidt ${ }^{2}$, Christian Duriez ${ }^{2}$ \\ ${ }^{1}$ Oscar Lambret Hospital, Lille, France \\ ${ }^{2}$ INRIA - University of Lille 1, France
}

\begin{abstract}
We introduce a new methodology for semi-automatic deformable registration of anatomical structures, using interactive inverse simulations. The method relies on non-linear real-time Finite Element Method (FEM) within a constraint-based framework. Given a set of few registered points provided by the user, a real-time optimization adapts the boundary conditions and(/or) some parameters of the FEM in order to obtain the adequate geometrical deformations. To dramatically fasten the process, the method relies on a projection of the model in the space of the optimization variables. In this reduced space, a quadratic programming problem is formulated and solved very quickly. The method is validated with numerical examples for retrieving Young's modulus and some pressures on the boundaries. Then, we apply the approach for the registration of the parotid glands during the radiotherapy of the head and neck cancer. Radiotherapy treatment induces weight loss that modifies the shape and the positions of these structures and they eventually intersect the target volume. We show how we could adapt the planning to limit the radiation of these glands.
\end{abstract}

\section{Introduction}

Radiation therapy (or radiotherapy) is one of the possible treatments for head and neck cancers. It uses high-energy X-rays to destroy the cancer cells. A treatment is established by using a treatment planning system (TPS) [1] which combines patient medical images, radiation transport simulations and optimization algorithms in order to expose tumors to X-rays while sparing healthy structures. The treatment plan is then applied 5 days per week during 6 to 7 weeks in order to destroy the tumors. During these 7 weeks, the patient is exposed to several side effects, and in particular an important weight loss. This induces the motion and deformation on the anatomical structures and the TPS does not take into account these topography changes, which may lead to an important X-ray exposure of healthy tissues [2] [7]. For instance it is reported [3] that xerostomia (loss of saliva) is due to a higher (than planned) exposure of the parotid glands while treating throat cancers.

Our global motivation is to adapt the planning to account for morphological modifications in order to limit the radiation exposure of healthy structures. It 
has been shown that non-rigid registration and daily computation of the dose can reduce the radiations [7]. But the challenge remain on the registration method over the 7 -week period. While significant works have been achieved recently in the field of automatic non-rigid registration (the reader may refer to [4] for a recent survey), these methods do not provide an easy control for the physicians. These algorithms also lack robustness and consistency when images are very complex. On the contrary, dealing with manual segmentations and/or registrations is time-consuming for the physicians and is not a viable solution for adapting the planning along with the treatment of the patients. The Finite Element Method (FEM) has proven to be a good candidate for patient to patient registration problems. Our second motivation is to improve its current uses which exhibit several downsides. For instance, it is often based on applying forces (or displacements) through the analysis of images [4]. The physics of these boundary conditions is not correct (for a mechanical standpoint) as it mixes physics-based forces with image-based forces. Another issue relates to the quality of the image analysis: some boundary motion may not be captured due to a poor analysis of the image leading to an under-constrained system. Finally FEM-based registration is not predictive for the moment since forces cannot be inferred from the medical images. Our third and main motivation is to provide a registration tool that can be driven by the physician (for robustness problem) with a very simple interface, and with an easy and explicit (i.e. no black-box tool) control on the parameters that have been used for the registration.

These motivations have led to the development of a new semi-manual registration method. First, our method solves a real-time inverse problem on nonlinear FEM which has, to our knowledge, not been addressed before. This enables to retrieve some missing parameters of the deformations. Second, it provides a good control of the registration result by setting manually few registered points. Yet, using non-linear FEM, complex deformations may be captured, constrained in the optimization process and supervised interactively by the physician given the retrieved values of the parameters. Third, this method is applied in the context of radiotherapy in order to capture the non-rigid motion of the parotids due to weight loss. Fourth, a validation study conducted on 7 patients exhibit results whose quality is comparable with manual segmentation / registration while requiring significant less manpower. The decrease of patient exposure to radiations is also highlighted when using our results for adapting the TPS.

\section{Real-time optimization method}

This section details the formulation that is used for real-time inverse method on non-linear FEM (static Saint-Venant Kirchhoff model). It describes how it can be employed to estimate the external loads, pressures, displacements... (in the following we use the generic term of boundary conditions) that lead to a given deformation. To estimate the Young's modulus from an observed deformation, we need to know the intensity of the forces applied on the model (like any other inverse methods [5]). If this later requirement is not reached (only images of 
the deformation are available), we can estimate a ratio between parameters of different regions (like in [5]) and obtain the displacement (not the efforts) of the boundary conditions. This strategy will be used for the registration of the parotids described in the following section since medical images do not provide force measurements.

The static FEM used in this paper accounts for non-linear geometrical deformations and integrates over the structure a Hookean constitutive law. During each step $\mathrm{i}$ of the simulation, a linearization of the internal forces is computed:

$$
\mathbf{f}\left(\mathbf{x}_{\mathbf{i}}\right) \approx \mathbf{f}\left(\mathbf{x}_{\mathbf{i}-\mathbf{1}}\right)+\mathbf{K}\left(\mathbf{x}_{\mathbf{i}-\mathbf{1}}\right) d \mathbf{x}
$$

where $\mathbf{f}$ provides the volumetric internal stiffness forces at a given position $\mathbf{x}$ of the nodes, $\mathbf{K}(\mathbf{x})$ is the tangent stiffness matrix that depends on the actual position of the nodes and $d \mathbf{x}$ is the difference between consecutive positions in time $d \mathbf{x}=\mathbf{x}_{\mathbf{i}}-\mathbf{x}_{\mathbf{i}-\mathbf{1}}$. The lines and columns that correspond to fixed nodes are removed from the system to improve the condition number of the matrix $\mathbf{K}$. Static equilibrium (the sum of external and internal force equals to zero) is sought at each step:

$$
-\mathbf{K}\left(\mathbf{x}_{\mathbf{i}-\mathbf{1}}\right) d \mathbf{x}=\mathbf{p}+\mathbf{f}\left(\mathbf{x}_{\mathbf{i}-\mathbf{1}}\right)+\mathbf{J}^{T} \boldsymbol{\lambda}
$$

where $\mathbf{p}$ is the external forces (e.g. gravity) that are known and $\mathbf{J}^{T} \boldsymbol{\lambda}$ gathers the contributions of the Lagrange multipliers. Three types of multipliers are defined:

boundary multipliers $\boldsymbol{\lambda}_{b}$ : we use these constraints to describes the external efforts applied on the boundary conditions that creates the deformation. The location of the boundary conditions are supposed to be known, the directions of the effort $\mathbf{J}^{T}$ can be updated at each step, and $\boldsymbol{\lambda}_{b}$ is the unknown intensity of the efforts on boundaries. We can set (and update at each step $i$ ) an interval of potential values $\min \leq \boldsymbol{\lambda}_{b} \leq \max$.

parameters multipliers $\boldsymbol{\lambda}_{p}$ : these parameters influence the computation of the internal forces. We use a local derivation of the internal force by the parameter $p: \mathbf{f}(\mathbf{x}, p+d p) \approx \mathbf{f}(\mathbf{x}, p)+(\delta \mathbf{f}(\mathbf{x}, p) / \delta p) d p$. In that case, $\mathbf{J}^{T}=\delta \mathbf{f}(\mathbf{x}, p) / \delta p$ and $\boldsymbol{\lambda}_{b}=d p$ is the variation of the parameter. To keep the validity of the local derivation over a step $i$, we can set $-\epsilon \leq \boldsymbol{\lambda}_{p} \leq \epsilon$.

registration multipliers $\boldsymbol{\lambda}_{r}$ : set interactively by the user to do a local manual registration on a small number of points. Contrary to a lot of existing registration methods, we do not put any force (or energy) to the association of points or on image pixels so $\boldsymbol{\lambda}_{r}=0$. Even if null, these multipliers are useful to build the optimization problem.

Indeed, the next step consists of the projection of the FEM model equations into the constraint space: the size of matrix $\mathbf{K}$ is often very large so an optimization in the motion space would be computationally very expensive. Instead, using the Schur complement of the constraint problem, we do a projection that dramatically reduces the size of the research space.

Three steps are followed, that are standard in a constraint solving process: Step $\mathbf{I}$, a free configuration $\mathbf{x}_{\text {free }}$ of the deformable model is found by solving equation (2) with $\boldsymbol{\lambda}=0$. For the constraint defined on registration point, we 
compute a violation noted $\boldsymbol{\delta}_{r}^{\text {free }}$ which provides a vector between the registered position of the points and the position given during the free motion. Step II: This step is central in the method. It consists in projecting the mechanics into the constraint space. As the constraints are the inputs (registered points) and outputs (parameters and efforts on boundary) of the inverse problem, we obtain the smallest possible projection space for the inverse problem:

$$
\boldsymbol{\delta}_{r}=\underbrace{\left[\mathbf{J}_{r} \mathbf{K}^{-1} \mathbf{J}_{p}^{T}\right]}_{\mathbf{W}_{r p}} \boldsymbol{\lambda}_{\boldsymbol{p}}+\underbrace{\left[\mathbf{J}_{r} \mathbf{K}^{-1} \mathbf{J}_{b}^{T}\right]}_{\mathbf{W}_{r b}} \boldsymbol{\lambda}_{\boldsymbol{b}}+\boldsymbol{\delta}_{r}^{\text {free }}
$$

$\boldsymbol{\delta}_{r}$ represents a vector between registered and actual positions of points chosen by the user. Then a Quadratic Programming (QP) problem is set by minimizing the norm of this vector.

$$
\begin{gathered}
\min \left(\frac{1}{2} \boldsymbol{\delta}_{r}^{T} \boldsymbol{\delta}_{r}\right)=\min \left(\frac{1}{2}\left[\begin{array}{c}
\boldsymbol{\lambda}_{\boldsymbol{p}} \\
\boldsymbol{\lambda}_{\boldsymbol{b}}
\end{array}\right]^{T}\left[\begin{array}{l}
\mathbf{W}_{r p}^{T} \\
\mathbf{W}_{r b}^{T}
\end{array}\right]\left[\begin{array}{ll}
\mathbf{W}_{r p} & \mathbf{W}_{r b}
\end{array}\right]\left[\begin{array}{c}
\boldsymbol{\lambda}_{\boldsymbol{p}} \\
\boldsymbol{\lambda}_{\boldsymbol{b}}
\end{array}\right]+\left[\begin{array}{c}
\boldsymbol{\lambda}_{\boldsymbol{p}} \\
\boldsymbol{\lambda}_{\boldsymbol{b}}
\end{array}\right]^{T}\left[\begin{array}{c}
\mathbf{W}_{r p} \\
\mathbf{W}_{r b}
\end{array}\right] \boldsymbol{\delta}_{r}^{\text {free }}\right) \\
\text { subject to } \min \leq \boldsymbol{\lambda}_{\boldsymbol{b}} \leq \max \text { and }-\epsilon \leq \boldsymbol{\lambda}_{\boldsymbol{p}} \leq \epsilon
\end{gathered}
$$

The size of the QP problem is much smaller than solving the problem in the motion space of equation 2, allowing to solve this problem in real-time. In practice, the QP matrix is always positive and definite if the size of $\boldsymbol{\delta}_{r}$ is greater than the number of optimized values in $\boldsymbol{\lambda}_{\boldsymbol{p}}$ and $\boldsymbol{\lambda}_{\boldsymbol{b}}$. During Step III, the final configuration of the deformable model is corrected by using the value of the constraint response using $\mathbf{x}_{i}=\mathbf{x}_{\text {free }}+\mathbf{K}^{-1}\left(\mathbf{J}_{p}^{T} \boldsymbol{\lambda}_{p}+\mathbf{J}_{b}^{T} \boldsymbol{\lambda}_{b}\right)$. In practice, we use a $L D L^{T}$ factorization of the matrix $\mathbf{K}$ and not $\mathbf{K}^{-1}$ during the computation.

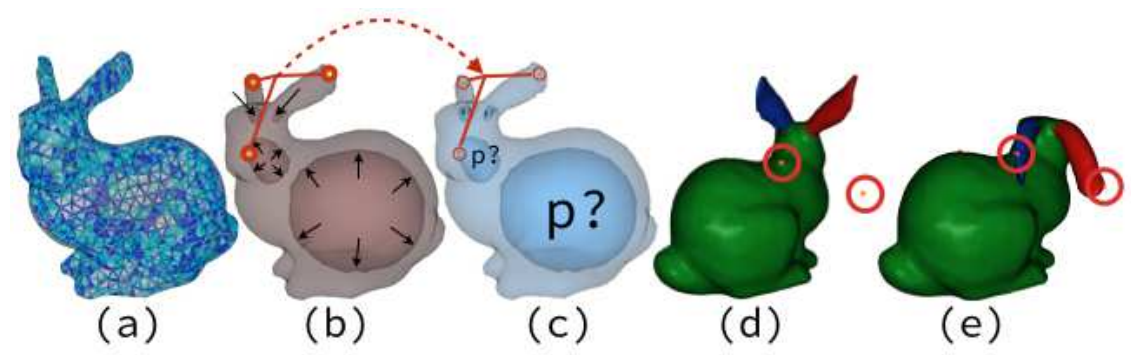

Fig. 1: Numerical validation: (a) Mesh composed of 5159 tetrahedra and 1673 points (b) forward simulation by setting pressures in 4 different cavities (c) inverse simulation by registration of 3 points. Young's modulus estimation under known gravity forces: (d) target points (highlighted in red) after setting 3 different Young's moduli (one color by Young's modulus), (e) resulting deformation once the modulus have been estimated.

Numerical validation: we present here a preliminary validation of the approach using numerical examples. In the first experiment, we place some cavities in a deformable object (courtesy of Stanford Computer Graphics Laboratory) and we apply different pressures in these cavities to create a deformation (Fig 
1(b)). We store the position of 3 points at equilibrium. Then we restart the simulation with no pressure in the cavity and register the 3 points using the inverse simulation approach(Fig 1(c)). The error in the pressure values is less than $1 \%$ and less than $5 \%$ with manual registration of the points. In the second example (Fig 1(d) and (e)), we follow the same methodology to retrieve 3 values of Young's Moduli using the fact that deformations were due to known gravity forces and error in the Young's moduli were less than $1 \%$.

\section{Application to adaptive radiotherapy}

In this section, we present the application of the method in the context of radiotherapy of the neck (throat cancer) where patient weight loss induces deformations of anatomical structures that are not taken into account in the treatment. Notably, the deformations of the parotids make them move towards the center of the neck and eventually intersect the main target volume of the therapy (see figure 2). Consequently parotids may be irradiated more than initially planned leading to xerostomia (20\% of patients).

Yet, with a robust registration performed just before the therapy, the planning could be adjusted. We emphasize that the parotids are poorly visible in the images, making the automatic registration not robust. With our inverse approach, the radiotherapist can use his expertise (knowledge of anatomy and meaningful parameters used in medical studies) to perform the registration and he/she will have a direct control of the parameters used for the registration.

For instance [6], studied the parotids deformation by performing CT scans of the patient three times a week during the entire treatment. Form that study, the deformation of the parotids is characterized by two parameters: the volume loss of the parotids and the motion of its center of mass (due to the volume loss of other structures). Parotids deformations observed are large (more than $30 \%$ the size of the structure) and can not be captured with linear models. These two parameters are introduced in our registration method and detailed in the next paragraph. The parotids are modeled with FEM models of about 650 tetrahedra and 200 nodes. The points that are in contact with the mandible are fixed to be consistent with medical observations.
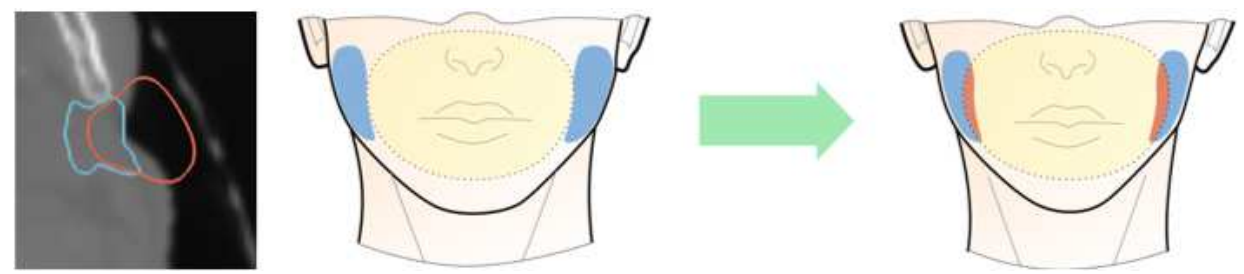

Fig. 2: Volume loss of parotids: (Left) segmentations of the parotids at weeks 1 (red) and 6 (blue). It is worth noticing the volume loss of the parotid as well as the motion of the center of mass. These two parameters have been used to characterize the deformation of parotids in [6]. (Right) Due to weight loss, parotids may intersect the target volume (in yellow). 
Boundary conditions retrieval: The first parameter (motion of the center of mass) is related to the volume loss of neighboring structures of the parotid leading to a global displacement. A constraint is built using a lumped (diagonal) mass matrix $\mathbf{M}$ on the FEM model. We can compute the total mass $m=\operatorname{trace}(\mathbf{M})$ Then, the position of the center of gravity $\mathbf{g}$ is computed as a weighted sum of the position $\mathbf{q}$ of each vertex of the mesh $\mathbf{g}=\sum_{j=0}^{N}\left(m_{j j} /(m+N)\right) \mathbf{q}$. Where $m_{j j}$ is the mass on node $j$. This linear relation provides the construction of the Jacobian: $\mathbf{g}=\mathbf{J}_{g} \mathbf{q}$. Practically, the motion of the center of mass is only significant along axis $x$ (towards the center of the neck), so optimization is done only on this direction. In our experiments, we found that the results are very similar with and without directions $y$ and $z$. The projection $g_{x}=\mathbf{J}_{g, x} \mathbf{q}$ is used. QP formulation allows to constrain the direction of displacement of $g_{x}$ thanks to a unilateral constraint, so that the optimization never finds a solution where the parotid moves in the wrong direction (no volume increase of neighboring structures has been observed during the treatment).

A second constraint is built to apply a volume loss to the parotid. The constraint is built so that if the tissue has a homogeneous properties, an homogeneous volume loss is observed. We compute a weighted normal at each point of the surface of the mesh that is proportional to the surface area of its neighboring triangles. Then, we apply a geometric diffusion of these normals inside the volume. So, at each point, we have a vector that provides the direction of the constraint. We put these directions in the Jacobian vector of the constraint $\mathbf{J}_{v}$. We introduce a unilateral condition in the QP so that the constraint can only reduce the volume. We can concatenate Jacobian vectors $\mathbf{J}_{g, x}$ and $\mathbf{J}_{v}$ to obtain the Jacobian matrix of the boundary condition $\mathbf{J}_{b}$. Sometimes, the volume loss of the parotids is not homogeneous. In that case, we divide the parotid in 3 regions, along the principal geometrical axis of the structure and non-uniform volume loss is computed in the optimization.

Interactive inverse simulation for planning update Our application starts with the geometrical models of the parotids that have been segmented during the initial planning and a CT image of the patient, after several weeks of therapy.
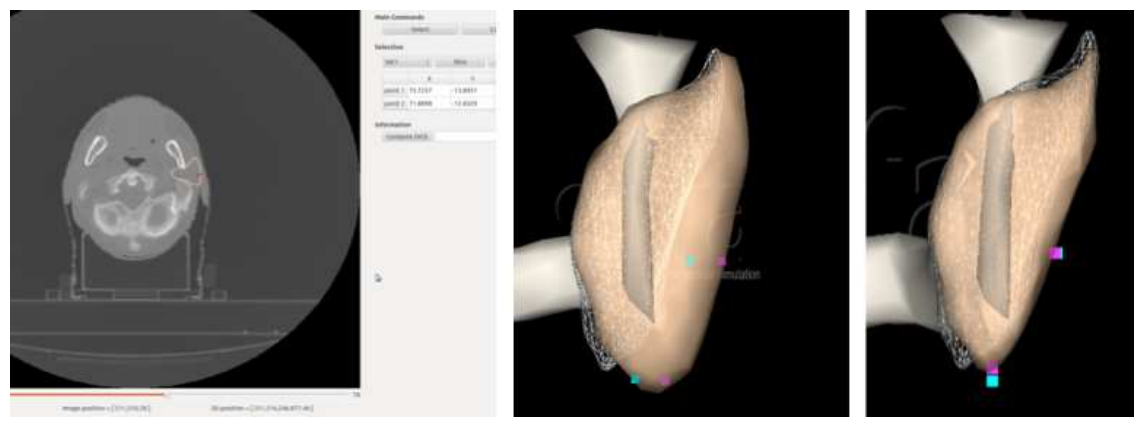

Fig. 3: Registration of the parotid deformations: (left) user interface that allows to select $2 \mathrm{D}$ points to be registered. (middle) in purple points to be registered on the targeted points (blue). (right) parotid deformation after our inverse simulation. 
First, a rigid registration between the meshes and the image is performed using the position of mandible bone. Then, the physician is asked to pick several points on the surface of the mesh and register them on the image (see figure 3 ). As this registration is done on a $2 \mathrm{D}$ slice of the $3 \mathrm{D}$ image, each registered point creates a $2 \mathrm{D}$ constraint. 3D registration is achieved when the user places points on different slices. The inverse simulation starts when the number of registered directions is superior to the number of unknowns. Practically, a maximum of 5 values are retrieved during the optimization, so 3 registered points (since each register point induces two constraints) are sufficient. Obviously to improve the precision of the registration, more parameters or boundary condition values can be optimized, but the user will have to register more points.

Validation by comparison with ground truth segmentation: the current medical routine does not adapt the treatment since it involves the manual segmentation of the structures -which is time and manpower consuming- and the computation of the new planning. However our method can dramatically reduce the time required to adapt the planning while achieving comparable accuracy to manual segmentation. We tested our approach on a ground truth set of 7 patient datasets that contains the 3D images of the CT scan done every week of the therapy (total: $7^{*} 6$ images). Comparison between manual segmentations of the parotids (performed by the radiologists) and our method is achieved on all available images (42) by computing the DICE coefficient. A single dataset (6 images) has been manually segmented by two radiologists and an average DICE coefficient of 0.7 has been computed to serve as a reference for the quality of our method. On these data, our method can be executed very quickly (completion of the registration is done in a single minute) with respect to a full manual segmentation making it compatible with the time constraints of a clinical routine. The graph 4 (left) illustrates that the parotids deformation is significant and second that our method exhibits really good similarity compared to manual segmentation.

Efficiency of the whole approach: For a first evaluation of the method, we selected a dataset of a patient: the deformations were important and the parotids were
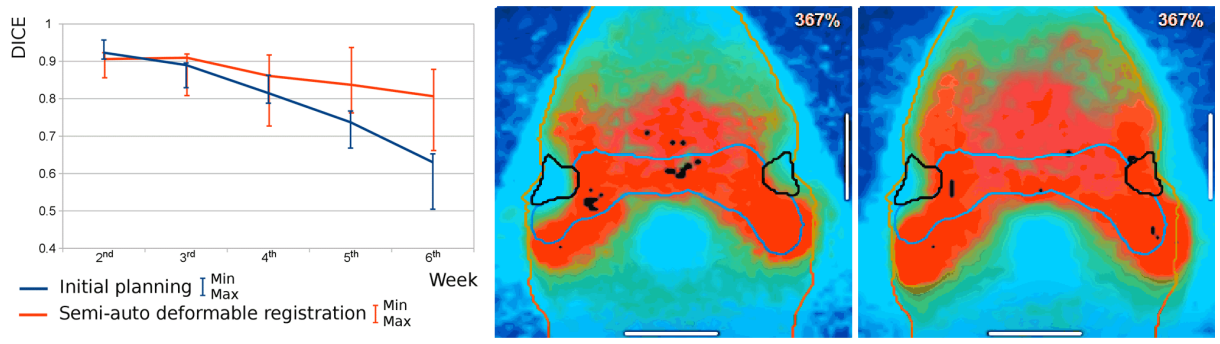

Fig. 4: Validation: (left) similarity between the initial segmentation and ground truth geometry in blue curve illustrates the deformation of the parotids (DICE decreasing), the red curve exhibits the good similarities between our results and the ground truth geometry; (middle) planning adaptation using our registration vs no planning adaptation (right). The measured radiation is much lower when the planning is adapted. 
not infiltrated by the tumor (therefore out of the target volume). We focus on the last session of the therapy and particularly at the irradiation map of the parotids without considering planning adaptation 4 (right) and with planning adaptation using our method to register the right parotid (middle). The resulting maps from the TPS show that the irradiation of the right parotid is significantly reduced and may limit the appearance of irradiation side-effects.

\section{Conclusion}

In this paper we presented a new method for patient to patient registration, based on inverse real-time simulation and an interactive manual registration of a few set of points. This method allows for a good control of the physician on the registration results, which is critical in applications such as radiotherapy. The method could have many other applications including parameter estimation for biomechanics of soft tissues or physics based registration. In future work, the results provided by the inverse method will be confronted to real measurements (for the Young's Modulus for instance) and it will be extended to other elastic parameters (Poisson's ratio). For the radiotherapy application, we will extend the approach to the registration of all the structures around the tumor, instead of only considering the parotids. Moreover, we will investigate more quantitatively how much the therapist can compensate the bad quality of images from cone beam CT (routinely aquired before the treatment) by her/his knowledge and interpretation using our method.

Acknowledgements: Authors would like to thank Pierre Jannin for his advice on validation. The project had the financial support of ANR JCJC Simi3 (Ideas), Oscar Lambret Hospital and Inria Lille Nord-Europe research centre.

\section{References}

1. Webb, S.: The physical basis of IMRT and inverse planning. The British Journal of Radiology, 76, 678-689 (2003).

2. Nelms, B. and Tomé, W. and Robinson, G. and Wheller, J.: Variations in the contouring of organs at risk: test case from a patient with oropharyngeal cancer. Intl Journal of Radiation Oncology,Biology,Physics, 82, 368-378 (2012).

3. Dirix, P. and Nuyts, S. and Van den Bogaert W.: Radiation-induced xerostomia in patients with head and neck cancer. Cancer, 107(11):2525-34 (2006).

4. Crum, W.R. and Hartkens, T. and Hill, D.L.G.: Non-rigid image registration: theory and practice. British Institute of Radiology (2014).

5. Lee H-P, Foskey M., Niethammer M., Krajcevski, P., Lin M.: Simulation-based joint estimation of body deformation and elasticity parameters for medical image analysis. IEEE Transactions on Medical Imaging, 31, 2156-2168 (2012).

6. Barker JL et al., Quantification of volumetric and geometric changes occurring during fractionated radiotherapy for head-and-neck cancer using an integrated CT/linear accelerator system, Intl Jnl of Rad. Oncology Biology Physics (2004).

7. Veiga, C. et al, Toward adaptive radiotherapy for head and neck patients: Feasibility study on using CT-to-CBCT deformable registration for dose of the day calculations. Medical physics, 41(3), 031703. (2014) 